\title{
Interlayer Interaction and Electronic Screening in Multilayer Graphene Investigated with Angle-Resolved Photoemission Spectroscopy
}

\author{
Taisuke Ohta, ${ }^{1,2}$ Aaron Bostwick, ${ }^{1}$ J. L. McChesney, ${ }^{1,3}$ Thomas Seyller, ${ }^{4}$ Karsten Horn, ${ }^{2}$ and Eli Rotenberg ${ }^{1}$ \\ ${ }^{1}$ Advanced Light Source, Lawrence Berkeley National Laboratory, Berkeley, California 94720, USA \\ ${ }^{2}$ Fritz-Haber-Institut der Max-Planck-Gesellschaft, Berlin, Germany \\ ${ }^{3}$ Montana State University, Bozeman, Montana 59717, USA \\ ${ }^{4}$ Lehrstuhl für Technische Physik, Universität Erlangen-Nürnberg, Erlangen, Germany
}

(Received 5 December 2006; published 16 May 2007)

\begin{abstract}
The unusual transport properties of graphene are the direct consequence of a peculiar band structure near the Dirac point. We determine the shape of the $\pi$ bands and their characteristic splitting, and find the transition from two-dimensional to bulk character for 1 to 4 layers of graphene by angle-resolved photoemission. By detailed measurements of the $\pi$ bands we derive the stacking order, layer-dependent electron potential, screening length, and strength of interlayer interaction by comparison with tight binding calculations, yielding a comprehensive description of multilayer graphene's electronic structure.
\end{abstract}

DOI: 10.1103/PhysRevLett.98.206802

Much recent attention has been given to the electronic structure of multilayer films of graphene, the honeycomb carbon sheet which is the building block of graphite, carbon nanotubes, $\mathrm{C}_{60}$, and other mesoscopic forms of carbon [1]. Recent progress in synthesizing or isolating multilayer graphene films [2-4] has provided access to their physical properties, and revealed many interesting transport phenomena, including an anomalous quantum Hall effect [5,6], ballistic electron transport at room temperature [7], micronscale coherence length [7,8], and novel many-body couplings [9]. These effects originate from the effectively massless Dirac fermion character of the carriers derived from graphene's valence bands, which exhibit a linear dispersion degenerate near the so-called Dirac point energy $E_{D}[10]$.

These unconventional properties of graphene offer a new route to room temperature, molecular-scale electronics capable of quantum computing $[6,7]$. For example, a possible switching function in bilayer graphene has been suggested by reversibly lifting the band degeneracy at the Fermi level $\left(E_{F}\right)$ upon application of an electric field $[11,12]$. This effect is due to a unique sensitivity of the band structure to the charge distribution brought about by the interplay between strong interlayer hopping and weak interlayer screening, neither of which is currently well understood $[13,14]$.

In order to evaluate the interlayer screening, stacking order, and interlayer coupling, we have systematically studied the evolution of the band structure of one to four layers of graphene using angle-resolved photoemission spectroscopy (ARPES). We demonstrate experimentally that the interaction between layers and the stacking sequence affect the topology of the $\pi$ bands, the former inducing an electronic transition from 2D to 3D (bulk) character when going from one layer to multilayer graphene. The interlayer hopping integral and screening length are determined as a function of the number of graphene layers by exploiting the sensitivity of $\pi$ states to the Coulomb potential, and the layer-dependent carrier concentration is estimated.
PACS numbers: 73.21. $-\mathrm{b}, 73.22 .-\mathrm{f}, 73.90 .+\mathrm{f}, 79.60 .-\mathrm{i}$

The films were synthesized on $n$-type (nitrogen, $1 \times$ $10^{18} \mathrm{~cm}^{-3}$ ) $6 \mathrm{H}-\mathrm{SiC}(0001)$ substrates (SiCrystal AG) that were etched in hydrogen at $1550{ }^{\circ} \mathrm{C}$. Annealing in a vacuum first removes the resulting silicate adlayer and then causes the growth of the graphene layers between $1250{ }^{\circ} \mathrm{C}$ to $1400^{\circ} \mathrm{C}$ [15]. Beyond the first layer, the samples have a \pm 0.5 monolayer thickness variation; the band structures of different thicknesses were extracted using the method of Ref. [11]. ARPES measurements were conducted at the Electronic Structure Factory end station at beam line 7.01 of the Advanced Light Source (ALS), equipped with a Scienta R4000 electron energy analyzer. The samples were cooled to $\sim 30 \mathrm{~K}$ by liquid $\mathrm{He}$. The photon energy was $94 \mathrm{eV}$ with the overall energy resolution of $\sim 30 \mathrm{meV}$ for Figs. 1 and 2(a)-2(d).

The band structures of a single [Fig. 1(a)] and a bilayer [1(b)] of graphene are reflected in their photoemission intensity patterns as a function of $k_{\|}$. The data are compared with the scaled density functional theory (DFT) band structures (see below) of free standing graphene layers

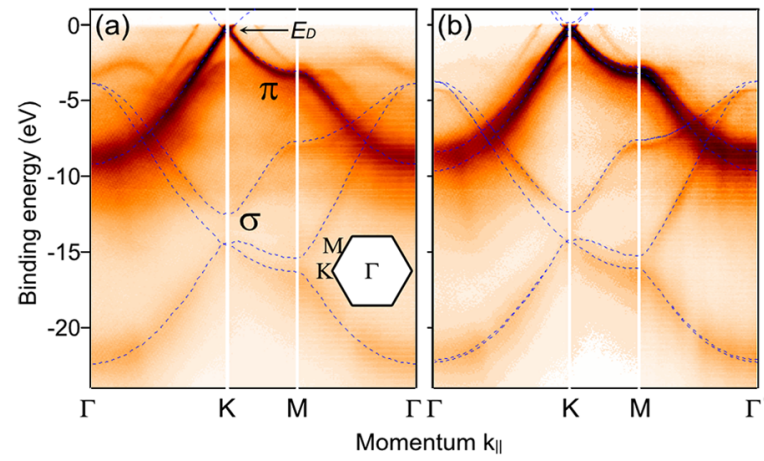

FIG. 1 (color online). Photoemission images revealing the band structure of (a) single and (b) bilayer graphene along high symmetry directions $\Gamma-K-M-\Gamma$. The dashed (blue) lines are scaled DFT band structure of freestanding films [16]. Inset in (a) shows the 2D Brillouin zone of graphene. 


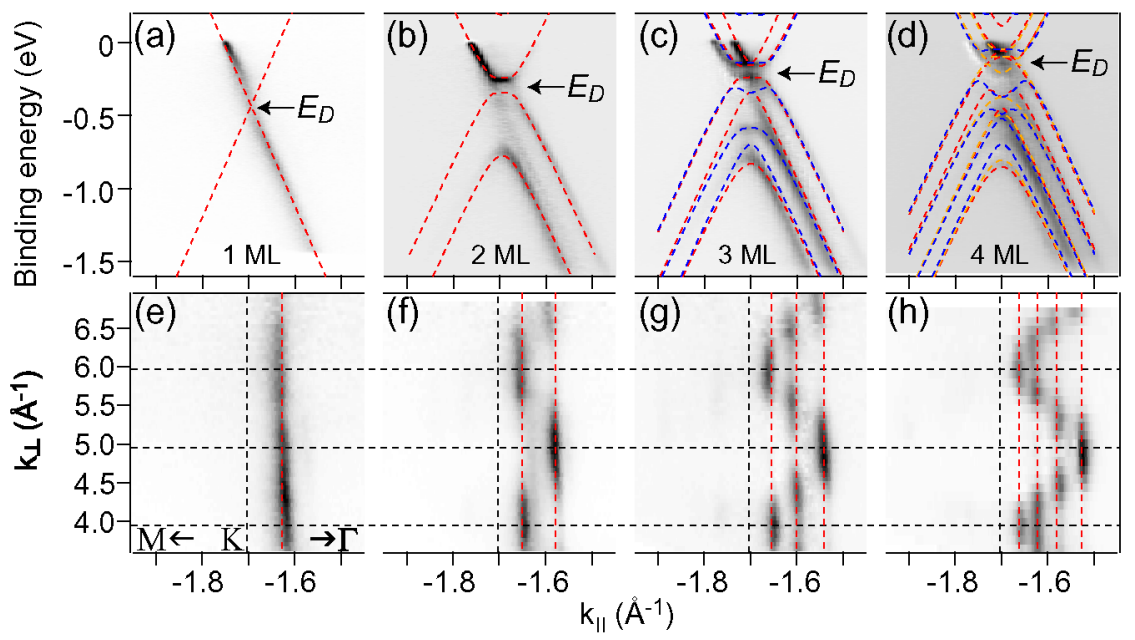

FIG. 2 (color online). (a)-(d) The $\pi$ and $\pi^{*}$ bands near $E_{F}$ for 1-4 graphene layers, respectively. $k_{\|}=-1.703 \AA^{-1}$ corresponds to the $K$ point. The $\Gamma$ point is at $k_{\|}=0 \AA^{-1}$, while the $M$ point is at $-2.555 \AA^{-1}$. The dashed lines are from a calculated tight binding band structure, with band parameters adjusted to reproduce measured bands. Red and orange (light gray) lines are for Bernal-type $(A B A B$ and $A B A C)$ stackings, while blue (dark gray) lines are for rhombohedral-type stackings. (e)-(h) Photoemission intensity oscillation of $\pi$ bands at $E_{F}-1 \mathrm{eV}$ as a function of $k_{\|}$and $k_{\perp}$ momentum for 1-4 layers graphene. The photoemission intensity is normalized by the angle integrated intensity between $E_{F}$ and $E_{F}-1.5 \mathrm{eV}$ for each photon energy.

[16]. We identify one $\pi$ and three $\sigma$ bands, with the $\pi$ band becoming split in the bilayer. At the $K$ point, the constant energy contour for the $\pi$ band is pointlike near $E_{F}$ in the monolayer $[9,11,17]$, similar to graphite at the bulk $H$ point $[18,19]$. Other features represent either underlying substrate states [especially in Fig. 1(a)] or replica bands due to diffraction from the underlying incommensurate interface structure of $\mathrm{SiC}[9,11,20,21]$.

The DFT bands are shifted to account for the Fermi level position, and expanded by $13 \%$ in energy to match the experimental total bandwidth. This scaling effectively incorporates many-body interactions not included in the theory, as shown in an earlier ARPES study of graphite [22]. The experimental bandwidths of the films are in close agreement with those of graphite [22] and thick graphene multilayers [20].

The $\pi$ bands near $E_{D}$ exhibit a complex structure due to interlayer interactions [11]. Detailed photoemission intensity maps near $E_{F}$ are shown in Figs. 2(a)-2(d) for $N=$ 1-4 graphene layers. The measured $\pi$ bands are suppressed on one side of the Brillouin zone due to interference effects between the two equivalent sublattices [23]. The number of $\pi$ bands increases with the number of layers due to interlayer interaction, clearly seen away from $E_{D}$, where the Coulomb potential of each layer does not play a major role (see below). The splitting between the highest and lowest $\pi$ bands increases with the number of layers, and for quadlayer graphene [Fig. 2(d)] it is close to that of bulk graphite, $\sim 0.7 \mathrm{eV}[18,24]$. There is a gap between the $\pi$ and $\pi^{*}$ bands in the bilayer [Fig. 2(b)] due to the inequivalent on-site Coulomb potentials in each layer [11].

The $\pi$ bands may be modeled by a tight binding (TB) calculation that takes into account the different stacking sequences and on-site potential energies, with a Hamiltonian generalized from Refs. $[12,25,26]$ as

$$
\begin{gathered}
\mathcal{H}=\left(\begin{array}{cccccc}
\alpha_{1} & \beta_{0} & & & & \\
\beta_{0}^{T} & \alpha_{2} & \beta_{s} & & & \\
& \beta_{s}^{T} & \alpha_{3} & \beta_{0} & & \\
& & \beta_{0}^{T} & \alpha_{4} & \beta_{s} & \\
& & & \beta_{s}^{T} & \ddots & \\
& & & \multicolumn{2}{c}{\alpha_{N}}
\end{array}\right), \\
\alpha_{i}=\left(\begin{array}{cc}
E_{i} & v \pi^{\dagger} \\
v \pi & E_{i}
\end{array}\right), \quad \beta_{s}=\gamma_{1}\left(\begin{array}{cc}
0 & s \\
1-s & 0
\end{array}\right) .
\end{gathered}
$$

Here $E_{i}$ is the on-site Coulomb energy for layer $i, \pi=$ $p_{x}+i p_{y}, \gamma_{1}$ is the interlayer hopping integral, $v$ is the band velocity, and $s=0$ for Bernal $(A B A \ldots)$ and 1 for rhombohedral $(A B C \ldots)$ stacking. The Hamiltonian operates on the layer subspace $i=(1,2, \ldots, N)$ while the $2 \times 2$ operators $\alpha$ and $\beta$ act on the $(A, B)$ sublattice sites of the same or adjacent layers, respectively. The energy scale was defined such that $E_{F}=0$ and the Dirac crossing energy $E_{D}=\operatorname{Tr} \mathcal{H} / 2 N$. The Hamiltonian can be readily generalized to arbitrary stacking orders (e.g., $A B A C$ ) by suitable rearrangements of the coupling operators $\beta_{s}$.

It is well known that $A B A$ and $A B C$ stackings for graphene are energetically close, and stacking faults are commonly found in highly ordered pyrolytic graphite [27]. For samples with mixed stacking, we assumed that the on-site Coulomb potentials do not depend on stacking sequence, and that they change monotonically across the film with the same sign [14]. Given that the potential must decay in a monotonic fashion, this considerably constrains the TB parameters.

For trilayer graphene [Fig. 2(c)], we find two sets of $\pi$ bands resulting from different stacking sequences. The red (light gray) and blue (dark gray) dashed lines indicate TB 
TABLE I. TB band parameters to reproduce measured band structure for $N=1-4$ layers graphene (present work) and $N=$ $\infty$ (graphite, Refs. [18,24]). The electron density $n$ is measured in $10^{-3}$ electron per $2 \mathrm{D}$ unit cell.

\begin{tabular}{ccccccccc}
\hline \hline$N$ & $v$ & $n$ & $E_{D}$ & $E_{1}$ & $E_{2}$ & $E_{3}$ & $E_{4}$ & $\gamma_{1}$ \\
\hline & $\left(10^{6} \mathrm{~m} / \mathrm{s}\right)$ & & & $(\mathrm{eV})$ & & \\
\hline 1 & 1.10 & 6.0 & -0.44 & -0.44 & & & & \\
2 & 1.05 & 8.1 & -0.30 & -0.35 & -0.24 & & & 0.48 \\
3 & 1.06 & 8.0 & -0.21 & -0.34 & -0.16 & -0.14 & & 0.44 \\
4 & 1.06 & 7.7 & -0.15 & -0.37 & -0.10 & -0.06 & -0.05 & 0.44 \\
$\infty$ & 0.91 & & & & & & & $\sim 0.35$ \\
\hline \hline
\end{tabular}

bands with Bernal and rhombohedral stackings, respectively. The effect of different stackings is most apparent for the middle $\pi$ band near $E_{F}-0.5 \mathrm{eV}$, but is also seen in the upper and lower $\pi$ bands. The situation is somewhat different for the quadlayer shown in Fig. 2(d). The red and orange (light gray) dashed lines are for Bernal-type stackings, $A B A B$ and $A B A C$, respectively. The blue (dark gray) dashed lines are for rhombohedral-type stackings, $A B C A$ and $A B C B$. Strictly speaking, the $A B C B$ sequence does not fall into the category of rhombohedral stacking; however, it is equivalent to the $A B C A$ sequence within the nearest neighbor TB model employed here. Four $\pi$ bands are well reproduced by assuming Bernal-type stackings, with the additional weak interspersed photoemission intensity suggesting minor contributions from rhombohedral-type stackings. The dominance of Bernal-type stackings for a quadlayer contrasts with the coexistence of Bernal and rhombohedral stackings in the trilayer, and suggests the role of the second nearest neighbor in stabilizing Bernal stacking in bulk graphite.

The tight binding parameters (Table I) reveal important details of the interlayer coupling and screening of graphene. Notice that our interlayer hopping integrals $\gamma_{1}$ for multilayer graphene are significantly larger than those of bulk graphite. In bilayer graphene, the interlayer hopping integral is reported to increase upon increasing carrier concentration [11]. We postulate that the larger interlayer hopping integral is similarly brought about by the higher carrier concentrations than that of graphite.

The $\pi$ bands of a single layer do not display a dispersion with out-of-plane electron momentum $k_{\perp}$ since the latter is not a good quantum number in a pure $2 \mathrm{D}$ system. With each added layer, though, an additional band occurs [Figs. 2(a) $-2(d)$ ] because of interlayer interaction. This is reflected in a modulation of the normalized photoemission intensity with photoexcitation energy, shown for the $\pi$ bands at a binding energy of $1 \mathrm{eV}$ below $E_{F}$ in Figs. 2(e)2(h). The photoemission intensity was normalized by dividing out the total intensity between $E_{F}$ and $E_{F}-1.5 \mathrm{eV}$ along the $M-K-\Gamma$ direction at each $h \nu$. In this constantinitial-state plot, the change in intensity reflects the evolution of the transition matrix element. In these data, the normal component of the initial state electron wave vector $k_{\perp}$ inside the solid was derived on the basis of the free electron final state model through $k_{\perp}=$ $2 m / \hbar^{2} \sqrt{E_{\text {kin }}+V_{0}}$, with $E_{\text {kin }}$ the measured kinetic energy, an inner potential, $V_{0}=16.5 \mathrm{eV}[28,29]$, and assuming that $k_{\|}=-1.703 \AA^{-1}$ at the $K$ point. The intensity pattern in Figs. 2(e)-2(h), plotted as a function of $k_{\perp}$ and $k_{\|}$ for the different initial states, will eventually evolve into a continuous dispersion curve as the number of layers increases toward true 3D bulk graphite.

For single layer graphene, the photoemission cross section decays smoothly and monotonically (this slow variation is normalized out in the data of Fig. 2(e)]. For 2 to 4 layer graphene [Figs. 2(f)-2(h)], however, the photoemission cross sections oscillate with a periodicity of $\sim 2.0 \AA^{-1}$. This periodicity does not match with the reciprocal lattice vector of bulk graphite $\left(0.932 \AA^{-1}\right)$, which includes two graphene layers in the unit cell, but is close to the reciprocal interlayer distance of graphite $\left(1.86 \AA^{-1}\right)$. The slightly longer periodicity of the observed intensity oscillation may also imply a reduced interlayer distance in our films, although a precise assessment of the $k_{\perp}$ periodicity requires careful estimation of the inner potential. A reduction in the spacing for bilayer graphene was previously attributed to the increased screening with carrier concentration [11].

The intensity oscillations with $k_{\perp}$ for $N \geq 2$ are similar to oscillations reported for quantized thin film states [30,31] and enhanced photoemission cross section for surface states [32-34] near vertical transitions of the bulk crystal. The lack of such oscillations for monolayer graphene results not just from the trivial lack of overlying graphene layers, but also due to a notable lack of coupling to the substrate [21] consistent with the lack of $\pi$ bands in the underlying interface layer [20]. This suggests that the single layer graphene wave functions have ideal 2D character.

The total charge density $n$ was determined by measuring the $\pi$-band Fermi surface areas, and was found to be almost invariant with film thickness or stacking order (Table I); this explains the observed decrease of $E_{F}-E_{D}$ with the film thickness $N$ [Figs. 2(a)-2(d)]. The deviation of the carrier concentration for different thicknesses is due to the accuracy of the fitting procedure.

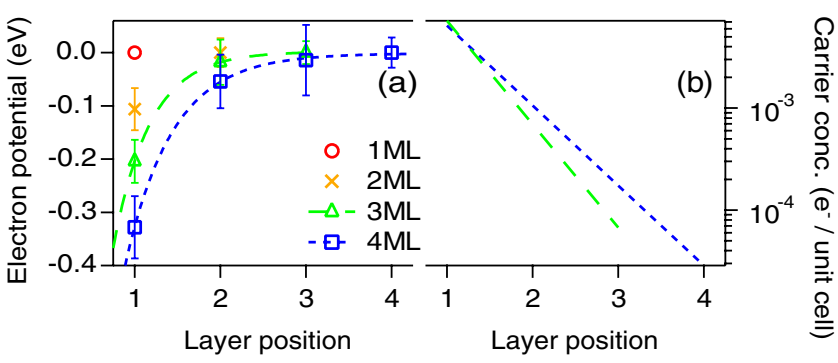

FIG. 3 (color online). Potential and carrier concentration profiles of the multilayer graphene as a function of the layer positions. The electron potentials are shifted in the way that the potential of the outermost graphene layer is at zero. 
The extracted on-site Coulomb potentials (Table I) may be used to estimate the screening length and the distribution of carrier concentrations. Shown in Fig. 3(a) is the onsite potential with the outermost layers' potentials aligned to the zero-reference level. The error bar is estimated from the energy width of each $\pi$ band at $k_{\|} \sim-1.65 \AA^{-1}$. Fitting the on-site potentials to a simple exponential decay allows a direct estimation of the interlayer screening lengths for three and four layers graphene, 1.4 and $1.9 \AA$, respectively. The estimated screening lengths are smaller than the reported value for graphite $(3.8-5 \AA[35,36])$, which has a much smaller carrier concentration than our films, thus a weaker electron screening.

Using Poisson's equation and the exponentially fitted potential profiles [Fig. 3(a)], the distribution of the charge density $n$ (Table I) across the layers can be estimated for $N=3$ and 4 graphene layers. An oscillation of the carrier concentration is predicted for graphene layers [14]. However, the predicted carrier concentration oscillations are a higher-order correction to our results considering the error bar on the experimentally determined potential profile. Shown in Fig. 3(b), the carrier concentration decreases by about 1 order of magnitude for each adjacent layer. For the reported multilayer graphene devices [8], the screening length is expected to be larger than the present case because of lower carrier concentration; therefore, the carrier concentration profile is expected to be less steep. This suggests that the carriers in less-doped multilayers are distributed across several layers.

While the electron potentials of a laterally confined surface state were previously extracted using a combination of ARPES and either scanning tunneling spectroscopy or iteratively evaluating wave functions $[37,38]$, the evaluation of the stacking order, potential, screening length, and carrier concentrations solely from ARPES band structure measurements is unique to the present study. The present analysis is only possible because the topology of the $\pi$ states is very sensitive to the on-site Coulomb potential, and because of the very high energy and momentum resolutions of the experiment.

In summary, we identify the $\pi$ band splitting due to interlayer interactions and different stacking sequences of the graphene layers. The interlayer interaction alters the character of the $\pi$ wave function from 2D in a single layer to a bulklike character in the multilayer graphene. Exploiting the sensitivity of the $\pi$ bands to the electron potential, the profiles of potential and carrier concentration in each layer and the screening length are estimated for three and four layer graphene. We find the interlayer hopping integral and screening length deviate significantly from those of graphite because of the altered carrier concentrations, which illustrate the unique electronic properties of graphene layers.

The authors thank K.V. Emtsev, J.M. Carlsson, F. Guinea, and A. Mascaraque for fruitful discussions. We are grateful to K. V. Emtsev and S. Reshanov for SiC prepa- ration. This work and ALS were supported by the U.S. Department of Energy, Office of Basic Sciences under Contract No. DE-AC02-05CH11231. T. O. and K. H. were supported by the Max Planck Society and the European Science Foundation under the EUROCORES SONS program.

[1] R. Saito, G. Dresselhaus, and M.S. Dresselhaus, Physical Properties of Carbon Nanotubes (Imperial College Press, London, 1998).

[2] K. S. Novoselov et al., Science 306, 666 (2004).

[3] Y. Zhang, J. P. Small, W. V. Pontius, and P. Kim, Appl. Phys. Lett. 86, 073104 (2005).

[4] C. Berger et al., J. Phys. Chem. B 108, 19912 (2004).

[5] K.S. Novoselov et al., Nature (London) 438, 197 (2005).

[6] Y. Zhang et al., Nature (London) 438, 201 (2005).

[7] C. Berger et al., Science 312, 1191 (2006).

[8] S. V. Morozov et al., Phys. Rev. B 72, 201401(R) (2005).

[9] A. Bostwick et al., Nature Phys. 3, 36 (2007).

[10] P. R. Wallace, Phys. Rev. 71, 622 (1947).

[11] T. Ohta et al., Science 313, 951 (2006).

[12] E. McCann, Phys. Rev. B 74, 161403(R) (2006).

[13] E. V. Castro et al., cond-mat/0611342.

[14] F. Guinea, cond-mat/0611185.

[15] I. Forbeaux, J. M. Themlin, and J. M. Debever, Phys. Rev. B 58, 16396 (1998).

[16] S. Latil and L. Henrard, Phys. Rev. Lett. 97, 036803 (2006).

[17] E. Rollings et al., J. Phys. Chem. Solids 67, 2172 (2006).

[18] S. Y. Zhou et al., Nature Phys. 2, 595 (2006).

[19] K. Sugawara et al., Phys. Rev. B 73, 045124 (2006).

[20] K. Emtsev et al., cond-mat/0609383.

[21] P. Mallet et al., cond-mat/0702406.

[22] C. Heske et al., Phys. Rev. B 59, 4680 (1999).

[23] E. L. Shirley, L. J. Terminello, A. Santoni, and F. J. Himpsel, Phys. Rev. B 51, 13614 (1995).

[24] J. C. Charlier, X. Gonze, and J. P. Michenaud, Phys. Rev. B 43, 4579 (1991).

[25] F. Guinea, A. H. Castro Neto, and N. M. R. Peres, Phys. Rev. B 73, 245426 (2006).

[26] E. McCann and V. I. Fal'ko, Phys. Rev. Lett. 96, 086805 (2006).

[27] G. Moos et al., Phys. Rev. Lett. 87, 267402 (2001).

[28] T. Ohta (unpublished).

[29] S. Y. Zhou, G. H. Gweon, and A. Lanzara, Ann. Phys. (N.Y.) 321, 1730 (2006).

[30] E. D. Hansen, T. Miller, and T.C. Chiang, J. Phys. Condens. Matter 9, L435 (1997).

[31] A. Mugarza et al., Phys. Rev. B 62, 12672 (2000).

[32] S. G. Louie et al., Phys. Rev. Lett. 44, 549 (1980).

[33] P. Hofmann et al., Phys. Rev. B 66, 245422 (2002).

[34] J. Lobo and A. Mascaraque, J. Phys. Condens. Matter 18, L395 (2006).

[35] L. Pietronero, S. Strassler, H. R. Zeller, and M. J. Rice, Phys. Rev. Lett. 41, 763 (1978).

[36] D. P. DiVincenzo and E. J. Mele, Phys. Rev. B 29, 1685 (1984).

[37] A. Mugarza, J. E. Ortega, F. J. Himpsel, and F. J. G. de Abajo, Phys. Rev. B 67, 081404(R) (2003).

[38] C. Didiot et al., Phys. Rev. B 74, 081404(R) (2006). 